\title{
COMPACTNESS IN LOCALLY COMPACT GROUPS ${ }^{1}$
}

\author{
BY RALPH HUGHES
}

Communicated by R. C. Buck, June 5, 1972

In [4], Glicksberg proved that every $\omega(G, \hat{G})$-compact subset of a LCA group $(G, \mathscr{T})$ is $\mathscr{T}$-compact. In this note three generalizations of this result are given (viz., Theorem 1, Theorem 3 and Theorem 4). In each case, a set is given two comparable topologies whose compacta are shown to coincide. It follows (with the weaker topology $T_{2}$ ) that sequential convergence also coincides in the two topologies.

THEOREM 1. Let $(G, \mathscr{T})$ be a locally compact $T_{2}$ group (not necessarily abelian) with $\hat{G}$ the continuous irreducible unitary representations of $G$. Then every $\omega(G, \hat{G})$-compact subset of $G$ is $\mathscr{T}$-compact. (For each $\pi$ in $\hat{G}$, the set of unitary operators on $H^{\pi}$ is given the weak operator topology.)

The separable metric case of Theorem 1 is proved first. A countable separating subfamily of $\hat{G}$ is shown to exist and an argument of Eberlein used to prove that every weakly compact set is weakly sequentially compact. Ernest proved [3, Corollary 4.5] that every weakly convergent sequence is $\mathscr{T}$-convergent, so this case of the proof is complete, since $\mathscr{T}$ is metric. The $\sigma$-compact case is shown to follow by virtue of the fact that a compact normal subgroup can be factored out to leave a separable metric quotient group. The general locally compact $T_{2}$ case is then established by showing (via irreducible positive definite functions) that every weakly compact set must lie in an open $\sigma$-compact subgroup of $G$.

The following theorem settles the question raised and partially answered by Bichteler in [1]. It is an immediate consequence of Theorem 1.

THEOREM 2. Let $\mathscr{T}_{1}, \mathscr{T}_{2}$ be locally compact Hausdorff topologies on a group $G$, which give rise to the same continuous irreducible unitary representations of $G$. Then $\mathscr{T}_{1}=\mathscr{T}_{2}$.

THEOREM 3. Let $(G, \mathscr{T})$ be a locally compact $T_{2}$ group and let $P(G)$ be the set of continuous positive definite functions on $G$. Then every subset of $P(G)$ compact in the topology of pointwise convergence is compact in the compact-open topology.

AMS (MOS) subject classifications (1970). Primary 22A99, 22D10, 43A35.

${ }^{1}$ This research comprises the author's doctoral dissertation written at the University of North Carolina at Chapel Hill under the direction of Professor Richard Iltis and Professor B. J. Pettis. 
Corollary. Let $X$ be a topological space such that $X \times G$ is a $k$-space, and let $f: X \times G \rightarrow C$ be a separately continuous function which is positive definite in the second variable. Then $f$ is jointly continuous.

(Note that Glicksberg's theorem mentioned above follows from Theorem 3 since, for $G$ abelian, $G \subseteq P(\hat{G})$.)

The next result, published by Corson and Glicksberg in [2], was obtained independently by us in 1968, with a somewhat different proof.

THEOREM 4. Let $G$ and $H$ be topological groups and suppose the closure of every compactly generated subgroup of $G$ is of second category in itself. Then every subset of the set $\operatorname{Hom}(G, H)$ (of continuous homomorphisms on $G$ to $H$ ) compact in the topology of pointwise convergence is compact in the compact-open topology.

COROLlary. Let $X$ be a topological space such that $X \times G$ is a $k$-space and let $f: X \times G \rightarrow H$ be a separately continuous function such that $f(x, g)$ is a homomorphism in $g$, for each $x \in X$. Then $f$ is jointly continuous.

In the proofs of both Theorem 3 and Theorem 4, a well-known theorem of Grothendieck [5, Theorem 5] on the equivalence of compactness and sequential compactness in certain function spaces is combined with a modified version of the Arzela-Ascoli theorem to allow consideration of sequential convergence. In Theorem 3, Haar measure and the Lebesgue Dominated Convergence Theorem are utilized, whereas Theorem 4 depends on a category theorem of Osgood [6, Theorem 9.5].

\section{REFERENCES}

1. K. Bichteler, Locally compact topologies on a group and the corresponding continuous irreducible representations, Pacific J. Math. 31 (1969), 583-593. MR 41 \# 394.

2. H. H. Corson and I. Glicksberg, Compactness in $\operatorname{Hom}(G, H)$, Canad. J. Math. 22 (1970), 164-170. MR 41 \# 382.

3. J. Ernest, $A$ strong duality theorem for separable locally compact groups, Trans. Amer. Math. Soc. 156 (1971), 287-307.

4. I. Glicksberg, Uniform boundedness for groups, Canad. J. Math. 14 (1962), 269-276. MR 27 \# 5856.

5. A. Grothendieck, Critères de compacité dans les espaces fonctionnels généraux, Amer. J. Math. 74 (1952), 168-186. MR 13, 857.

6. J. L. Kelley and I. Namioka, et al., Linear topological spaces, University Series in Higher Math., Van Nostrand, Princeton, N.J., 1963. MR 29 \# 3851.

Department of Mathematics, University of North Carolina at Chapel Hill, Chapel Hill, North Carolina 27514

Department of Mathematics, North Carolina Central University, Durham, NORTH CAROLINA 27707 